\title{
High susceptibility of wild Anopheles funestus to infection with natural Plasmodium falciparum gametocytes using membrane feeding assays
}

Cyrille Ndo ${ }^{1,2,3^{*}}$, Edmond Kopya ${ }^{1,4}$, Benjamin Menze-Djantio ${ }^{2,5}$, Jean Claude Toto ${ }^{1}$, Parfait Awono-Ambene', Gareth Lycett ${ }^{2}$ and Charles S. Wondji, ${ }^{2,5}$

\begin{abstract}
Background: Anopheles funestus is a major vector of malaria in sub-Saharan Africa. However, because it is difficult to colonize, research on this mosquito species has lagged behind other vectors, particularly the understanding of its susceptibility and interactions with the Plasmodium parasite. The present study reports one of the first experimental infections of progeny from wild-caught An. funestus with the $P$. falciparum parasite providing a realistic avenue for the characterisation of immune responses associated with this infection.

Methods: Wild-fed resting An. funestus females were collected using electric aspirators and kept in cages for four days until they were fully gravid and ready to oviposit. The resulting eggs were reared to adults F1 mosquitoes under insectary conditions. Three to five day-old An. funestus F1 females were fed with infected blood taken from gametocyte carriers using an artificial glass-parafilm feeding system. Feeding rate was recorded and fed mosquitoes were dissected at day 7 to count oocysts in midguts. Parallel experiments were performed with the known Plasmodium-susceptible An. coluzzii Ngousso laboratory strain, to monitor our blood handling procedures and infectivity of gametocytes.

Results: The results revealed that An. funestus displays high and similar level of susceptibility to Plasmodium infection compared to An. coluzzii, and suggest that our methodology produces robust feeding and infection rates in wild An. funestus progeny. The prevalence of infection in An. funestus mosquitoes was 38.52\% (range 6.25-100\%) and the median oocyst number was 12.5 (range 1-139). In parallel, the prevalence in An. coluzzii was $39.92 \%$ (range 6.85-97.5\%), while the median oocyst number was 32.1 (range 1-351).

Conclusions: Overall, our observations are in line with the fact that both species are readily infected with $P$. falciparum, the most common and dangerous malaria parasite in sub-Saharan Africa, and since An. funestus is widespread throughout Africa, malaria vector control research and implementation needs to seriously address this vector species too. Additionally, the present work indicates that it is feasible to generate large number of wild F1 infected An. funestus mosquitoes using membrane feeding assays, which can be used for comprehensive study of interactions with the Plasmodium parasite.
\end{abstract}

Keywords: Experimental infection, Anopheles funestus, Plasmodium falciparum, Membrane feeding

\footnotetext{
* Correspondence: cyrndo@yahoo.fr

${ }^{1}$ Malaria Research Laboratory, Organisation de Coordination pour la lutte contre les Endémies en Afrique Centrale (OCEAC), P.O. Box 288, Yaoundé, Cameroon

${ }^{2}$ Department of Vector Biology, Liverpool School of Tropical Medicine,

Pembroke Place, Liverpool L3 5QA, UK

Full list of author information is available at the end of the article
} 


\section{Background}

Despite recent progress in reducing malaria burden, it remains one of the most debilitating diseases in the tropical world with 198 million cases reported in 2013, leading to more than 500,000 deaths [1]. In sub-Saharan Africa, where about $90 \%$ of all malaria deaths occur, mosquitoes from Anopheles gambiae complex and Anopheles funestus group are the most efficient vectors [2-5], while $P$. falciparum is the most common and dangerous parasite responsible for most cases of severe malaria [1].

Current efforts to control or to eliminate malaria rely heavily on vector control interventions such as largescale distribution of long-lasting insecticide-treated nets (LLINs) and in some places through indoor residual house spraying (IRS) [6-8]. In parallel, research on new and innovative malaria control tools that could enhance the efficacy or complement existing approaches is needed. One of the approaches being pursued is the genetic replacement of vector populations with non-vectors in order to disrupt parasite transmission [9-11]. However, this requires first of all a good understanding of the complex and specific interactions between the Plasmodium parasites and its Anopheles vectors, notably the associated immune response.

Obtaining sufficient Plasmodium experimentally infected mosquitoes is the first and one of the most challenging steps of research on Anopheles-Plasmodium interactions. In An. gambiae which is easier to colonize and adapts well to feed on the artificial parafilm membrane, successful experimental infections are commonly performed using either the rodent $P$. berghei or cultured or recently field collected human $P$. falciparum parasites. This has allowed the identification of important mosquito factors that may prove to be favourable targets for novel interventions towards blocking malaria transmission [12-14].

If such progress has been achieved in $A n$. gambiae, little advance has been observed for the other major malaria vector An. funestus. It is known that An. funestus exhibits important differences to An. gambiae in term of its biology, genetics and ecology as shown by the difficulty of rearing and feeding this species in laboratory conditions [3]. Such difficulty has largely slowed the pace of research on this species, particularly its interactions with the Plasmodium parasite.

By collecting indoor resting gravid females and force them to lay eggs, it is now possible to generate large F1 colonies of $A n$. funestus for controlled experimentation $[15,16]$. This method already allowed study of insecticide resistance mechanisms throughout Africa [17-21]. Conversely, only two studies have investigated $A n$. funestus susceptibility to the rodent parasite $P$. berghei $[22,23]$, and none have been performed with the natural human parasite $P$. falciparum. Despite similarities between these two Plasmodium species, important differences in interactions with mosquito vectors have been reported $[14,24]$. This observation point out the necessity of following up discoveries in laboratory model systems with studies on natural parasitemosquito interactions, particularly in the perspective of investigating molecular bases of An. funestus immune response to Plasmodium infection.

This paper reports the first experiments in which first generation of wild-caught An. funestus mosquitoes are infected with natural $P$. falciparum gametocytes using an artificial blood feeding system.

\section{Methods}

\section{Mosquito collections}

Anopheles funestus mosquitoes were collected in Mebelong $\left(6^{\circ} 46^{\prime} \mathrm{N}, 11^{\circ} 70^{\prime} \mathrm{E}\right)$, a village situated in humid savannah region, about $350 \mathrm{~km}$ North of Yaoundé, the capital city of Cameroon. This site is characterised by a high density of mosquitoes resting indoors.

Resting mosquitoes were collected using electric aspirators (Rule In-Line Blowers, Model 240). In the field, $A n$. funestus mosquitoes were sorted from other Anopheles species based on morphological criteria [25, 26] and kept in paper cups for four days or more until they were fully gravid and ready to lay eggs. Once brought back in the insectary, females were allowed to oviposit individually using a forced egg-laying method [15]. After oviposition, all the carcasses were preserved in individual tubes containing desiccant for molecular identification [27, 28] and for enzyme-linked immunosorbent assay (ELISA), to assess Plasmodium infection rate. All other Anopheles species collected as well as An. funestus specimens that died before reaching the insectary were also preserved for ELISA, to identify blood meal source and assess Plasmodium infection rate according to $[29,30]$ and [31], respectively. Positive samples to ELISA were determined by reading the optical densities at $405 \mathrm{~nm}$ using an ELISA plate reader (BioTek ELx800, Swindon, UK).

We used the An. coluzzii Ngousso laboratory strain as a control sample to monitor for the effectiveness of blood handling procedure and infectivity of gametocytes during infection experiments. The Ngousso strain, routinely maintained at OCEAC, has been extensively used in experimental infection studies and is known to be well adapted to feed through artificial parafilm membrane and is highly susceptible to Plasmodium infection [32]. Anopheles funestus and An. coluzzii females were fed with blood from same gametocyte carriers to directly compare prevalence of infection.

\section{Identification of gametocyte carriers}

Parasitological surveys to detect gametocyte carriers were conducted during a high transmission period from March to June 2015. Children aged between 5 and 11 
were recruited at local schools of the locality of Okola $\left(4^{\circ} 01^{\prime} \mathrm{N}, 11^{\circ} 22^{\prime} \mathrm{E}\right)$, a rural area situated about $25 \mathrm{~km}$ from Yaoundé. Blood samples from each child enrolled in the study were screened by direct microscopic $(100 \times)$ visualization of Plasmodium parasites on thick blood smears stained with $10 \%$ Giemsa. Trophozoite density was determined using semi quantitative count (thick film) method following a semi-quantitative scale [33]. Gametocyte density was estimated by microscopy read against 500 leucocytes, assuming a standard white blood cell concentration of $8,000 / \mu \mathrm{l}[34]$.

\section{Experimental infections}

Gametocyte carriers were selected based on parasite density in blood after their haemoglobin content was first measured using Haemocue (HaemoCue AB, Angelholm, Sweden). Children with abnormally low haemoglobin rate were treated for anaemia and their blood was not used. For selected children, a volume of 3$4 \mathrm{ml}$ of blood was taken by venepuncture into heparinized tubes. The blood was immediately centrifuged at $37^{\circ} \mathrm{C}$ and the serum was replaced by the same volume of European AB serum (Sigma-Aldrich, Taufkirchen, Germany), prewarmed at the same temperature. The reconstituted blood was offered to mosquitoes immediately.

Groups of 80 to 100, 3-5-day-old F1 female mosquitoes were counted into paper cups covered with mosquito netting and starved $12 \mathrm{~h}$ before experiments. $450 \mu \mathrm{l}$ aliquots of reconstituted blood were placed in each glass feeder with a parafilm membrane maintained at $37^{\circ} \mathrm{C}$ using a circulating heating water bath (Fisher Scientific INC, Isotemp 4500H5P, Pittsburgh USA). Mosquitoes were allowed to feed for $45 \mathrm{~min}$, after which unfed and partially fed mosquitoes were removed. Mosquitoes were maintained in separate cups in the insectary at $26 \pm 2{ }^{\circ} \mathrm{C}$ and 70 to $80 \%$ relative humidity, with a constant supply of $10 \%$ sucrose and daily mortality was recorded. Part of fed mosquitoes were preserved in RNAlater for future transcriptomic analysis. The remaining ones were dissected at day 7 post-infection in a drop of $0.4 \%$ mercurochrome and stained midguts were examined for detection and quantification of oocysts under light microscopy $(40 \times)$ [35].

\section{Data analysis}

Parameters recorded included number of mosquitoes fed per batch, mortality to day 7 , number of mosquitoes infected at oocyst stage and number of oocysts per midgut. For each experiment, the median oocyst number was determined and mean oocyst number per midgut was calculated by dividing the total number of oocysts counted by the number of mosquitoes found infected after dissection.
Fisher's exact test was used to compare prevalence of infection between species. The median oocyst numbers were compared using the nonparametric MannWhitney test. Correlation between the prevalence of infection, the median number of oocyst and the gametocytemia was analysed using Spearman's correlation. All statistical analyses were performed using Graph Pad prism V.5 and P-values of 0.05 or less were considered as significant.

\section{Results \\ Mosquito population and rearing}

During eight days, a total of 1,918 mosquitoes belonging to three genera were collected in Mebelong. Anopheles mosquitoes were by far the most abundant representing $99.48 \%$ of mosquitoes, while Culex and Mansonia accounted for less than $1 \%$. Three Anopheles species were identified in the locality: An. funestus (s.l.) the main vector species $(n=1,831 ; 95.96 \%), A n$. gambiae (s.l.) ( $n=74 ; 3.88 \%)$ and An. pharoensis ( $n=3$; $0.16 \%)$. According to the molecular identification of a subsample of 890 mosquitoes, An. funestus (s.s.) (99.43\%) and An. leesoni (0.57\%) were the two members of the $A n$. funestus group present in the locality.

Anopheles funestus (s.s.) (hereafter An. funestus) survival rate was high with $73.73 \%(n=1,350)$ of mosquitoes reaching the insectary alive (after a $350 \mathrm{~km}$ journey in cooling box) and used for egg production. Similarly, high oviposition $(80.81 \%)$ and egg hatch $(85.43 \%)$ rates were recorded, indicating that large majority of mosquitoes were already inseminated at the time they were collected.

Preliminary ELISA analysis revealed that An. funestus actively transmits malaria in Mebelong, with anthropophilic and infection rates of $100 \%(n=100)$ and $3.7 \%$ $(n=656)$, respectively.

\section{Parasitological survey}

In total 1,091 children aged between five and 11 years were examined and the prevalence of detected asexual malaria was $40 \%$ in the survey population. Among the infected children, the result of blood smears ranged from $1+$ to $4+$, with the majority of them failing in the $1+(53.35 \%)$ and $2+(35.10 \%)$ groups [34] (Table 1$)$. According to this prevalence rate, our survey area could be classified as mesoendemic for malaria, although few children above ten years were included in the study [36, 37]. The prevalence of gametocyte carriers identified by blood smear in the total survey population examined was approximately $10 \%$.

\section{Plasmodium falciparum infection in An. funestus}

Fourteen infection experiments were carried out using blood from different gametocyte carriers. In total, 9,728 
Table 1 Summary of parasitological surveys. Data in parentheses represent prevalences. Gametocyte carriers were detected among children aged between 5 and 11 after screening of their blood samples by direct microscopic visualisation of Plasmodium parasites on thick blood smears stained with $10 \%$ Giemsa. Plasmodium trophozoite density was determined using semi quantitative count (thick film) method

\begin{tabular}{|c|c|c|c|c|c|c|c|}
\hline School & $\begin{array}{l}\text { Number } \\
\text { examined }\end{array}$ & TPF+ & TPF++ & TPF +++ & TPF ++++ & $\begin{array}{l}\text { Total tropozote } \\
\text { carriers }\end{array}$ & $\begin{array}{l}\text { Gametocyte } \\
\text { carriers }\end{array}$ \\
\hline Nkolyada & 74 & 22 & 15 & 1 & 0 & $38(51.35)$ & $4(5.41)$ \\
\hline Elig-Onana & 102 & 30 & 23 & 13 & 0 & $66(64.71)$ & $9(8.82)$ \\
\hline Okola G2 & 111 & 25 & 11 & 5 & 0 & $41(36.94)$ & $7(6.31)$ \\
\hline Zamengoé & 210 & 58 & 22 & 11 & 1 & $92(43.81)$ & $24(11.43)$ \\
\hline Nkolngock & 110 & 25 & 22 & 2 & 0 & $49(44.54)$ & $12(10.91)$ \\
\hline Ndangueng & 105 & 18 & 9 & 5 & 3 & 35 (33.33) & $8(7.62)$ \\
\hline Mvoua & 140 & 28 & 27 & 4 & 1 & $60(42.86)$ & $23(16.43)$ \\
\hline Levalombédé & 239 & 25 & 23 & 3 & 1 & $52(21.76)$ & $18(7.53)$ \\
\hline Total & 1,091 & 231 & 152 & 44 & 6 & 433 (39.69) & $101(9.26)$ \\
\hline
\end{tabular}

TPF levels: +, 1-10 trophozoites/100 microscopic thick film fields; ++, 1-10 trophozoites/10 microscopic thick film fields; +++, 1-10 trophozoites/single microscopic thick film field;,$++++>10$ trophozoites/single microscopic thick film field

Abbreviation: TPF trophozoite of $P$. falciparum

An. funestus females were given access to infected blood through an artificial parafilm membrane and 2,518 successfully fed, corresponding to a global feeding rate of $26 \%$ (Table 2). However, feeding rate significantly varied across experiments ranging from 18 to $47 \%$. Monitoring of the mortality to day 7 for nine batches of infected mosquitoes revealed a mortality rate between 0 and $69.23 \%$ (mean $38.05 \%$ ).

Plasmodium falciparum gametocytes were infective for mosquitoes in 11 (78.57\%) of 14 experiments with prevalence of infection ranging from 3.7 to $100 \%$ (mean $25.70 \%)$. A total of 1,165 oocysts were counted in all

Table 2 Summary of experimental infection parameters in An. funestus. Mosquitoes aged between 3 to 5 days were given infected blood through artificial parafilm membrane and midgut were dissected at day 7 post-infection for oocyst detection under light microscopy. Feeding rate was calculated by dividing the number of mosquitoes alive in cups after blood feeding by the number successfully fed. Prevalence of infection was calculated by dividing the number of mosquitoes infected at oocyst stage by the total number of mosquito dissected

\begin{tabular}{|c|c|c|c|c|c|c|c|c|}
\hline Experiment & $\begin{array}{l}\text { Gametocyte } \\
\text { density }\end{array}$ & $\begin{array}{l}\text { Feeding rate } \\
(\%)\end{array}$ & Dissected & Infected & $\begin{array}{l}\text { Prevalence of infection } \\
(\%)\end{array}$ & $\begin{array}{l}\text { Total oocyst } \\
\text { count }\end{array}$ & $\begin{array}{l}\text { Oocyst range } \\
\text { (Min-Max) }\end{array}$ & $\begin{array}{l}\text { Median oocyst } \\
\text { load }\end{array}$ \\
\hline$N^{0} 1$ & - & 24.52 & 32 & 11 & 34.37 & 45 & $1-15$ & 2.5 \\
\hline $\mathrm{N}^{\circ} 2$ & - & 19.10 & 36 & 22 & 61.11 & 162 & $1-18$ & 7 \\
\hline$N^{\circ} 3$ & - & 28.89 & 21 & 0 & 0 & 0 & - & - \\
\hline $\mathrm{N}^{\circ} 4$ & - & 25.25 & 32 & 2 & 6.25 & 4 & $1-3$ & 1 \\
\hline $\mathrm{N}^{\circ} 5$ & - & 23.78 & 39 & 6 & 15.38 & 9 & $1-4$ & 1 \\
\hline$N^{\circ} 6$ & - & 46.53 & 24 & 3 & 12.50 & 4 & $1-2$ & 1 \\
\hline $\mathrm{N}^{0} 7$ & - & 26.03 & 84 & 0 & 0 & 0 & - & - \\
\hline$N^{\circ} 8$ & - & 33.80 & 30 & 0 & 0 & 0 & - & - \\
\hline $\mathrm{N}^{\circ} 9$ & 96 & 31.38 & 59 & 42 & 71.19 & 241 & $1-16$ & 8 \\
\hline $\mathrm{N}^{\circ} 10$ & 32 & 19.19 & 43 & 14 & 32.56 & 20 & $1-2$ & 1 \\
\hline$N^{0} 11$ & 368 & 18.81 & 52 & 17 & 32.69 & 40 & $1-6$ & 2.5 \\
\hline $\mathrm{N}^{\circ} 12$ & 880 & 18.37 & 9 & 9 & 100 & 539 & $1-139$ & 60 \\
\hline $\mathrm{N}^{\circ} 13$ & 80 & 31.92 & 105 & 37 & 35.24 & 96 & $1-8$ & 4 \\
\hline $\mathrm{N}^{\circ} 14$ & 16 & 27.61 & 81 & 3 & 3.7 & 5 & $1-2$ & 1 \\
\hline All & - & 25.89 & 647 & 166 & 25.66 & 1165 & $1-139$ & 12.5 \\
\hline
\end{tabular}


infected midguts and the global median oocyst number was 12.5 (mean 7.02) (Table 2). Taking each experiment individually, the number of oocysts observed in a midgut ranged from 0 to 139 and median oocyst number varied from 1 to 60 (range for means 1.43-59.88).

The gametocyte density was determined for 6 successful experiments and ranged from 16 to 880 gametocytes/ $\mu \mathrm{l}$ of blood. There was a general trend showing that prevalence of infection and oocyst number in midguts increased with gametocyte density in blood samples (Fig. 1). However, the correlation was statistically significant only for oocyst number $\left(r_{s}=0.849, P=0.009\right)$ and prevalence of infection $\left(r_{s}=0.557, P=0.088\right)$.

\section{Comparative analysis of the susceptibility of An. funestus and $A n$. coluzzii to $P$. falciparum infection}

The same infected blood was given to An. funestus and An. coluzzii mosquitoes simultaneously in 12 parallel experiments for comparison. The feeding rate was two to three-fold higher in An. coluzzii lab strain compared to the wild An. funestus (Additional file 1: Table S1). Among the 11 experiments in which more than five mosquitoes were dissected for both species, ten $(90.91 \%)$ resulted in

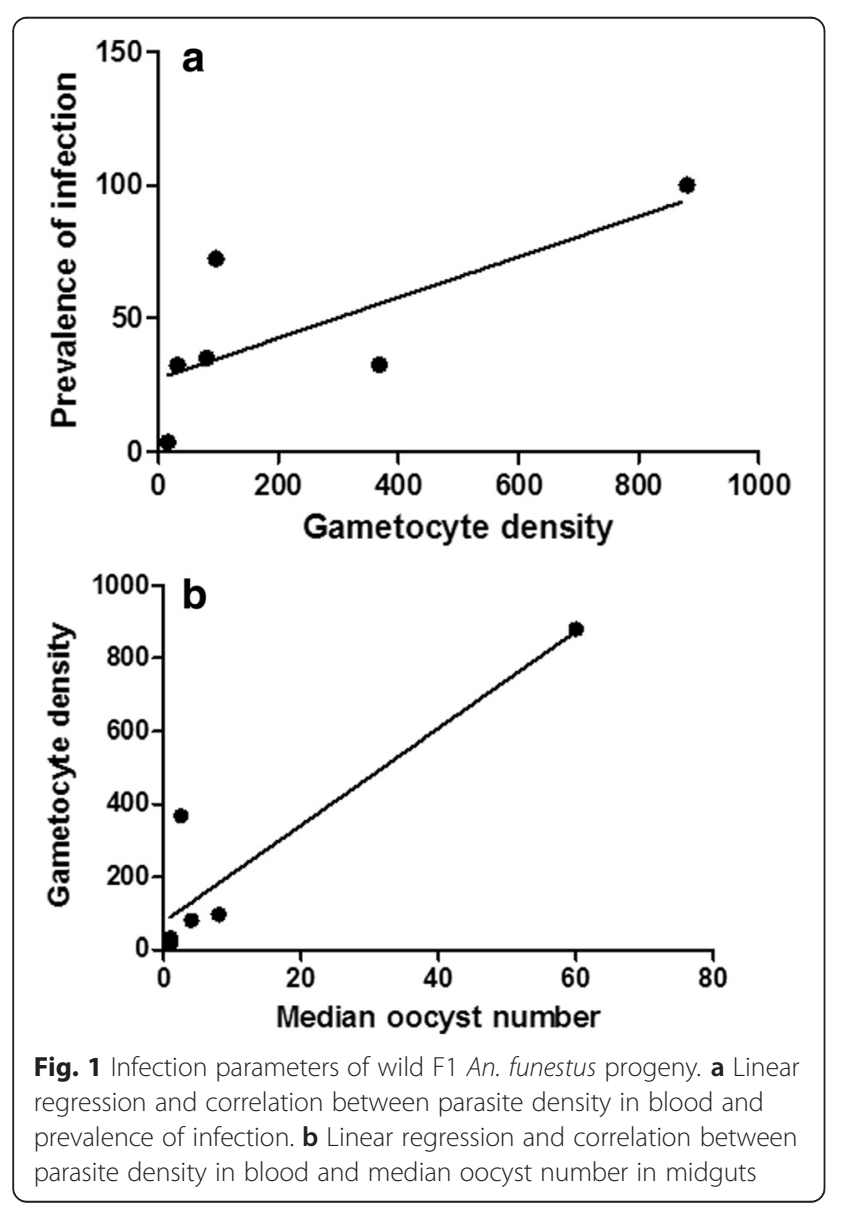

at least one infected mosquito in An. funestus compared to nine (81.82 \%) in An. coluzzii.

Overall, the two species showed high and comparable susceptibility to $P$. falciparum infection. Based on the nine experiments which were successful in both species, the prevalence of infection in An. funestus was $38.52 \%$ (range 6.25-100 \%) and the median oocyst number was 12.5 (mean 7.68; range 1-139). In parallel, the prevalence of infection in An. coluzzii was $39.92 \%$ (range 6.85-97.5\%), while the median oocyst number was 32.1 (mean 20.33; range 1-351) (Fig. 2). These two parameters did not vary significantly between the two species across experiments, with 2 exceptions (Additional file 1: Table S1).

\section{Discussion}

The susceptibility of An. funestus to Plasmodium infection remains relatively uncharacterised in laboratory conditions. The only two studies carried out to date were limited to the use of the rodent parasite $P$. berghei $[22,23]$. This model organism is more amenable than P. falciparum and has been instrumental in malaria research studies leading to important discoveries on mechanisms of parasite killing in Anopheles vectors [12-14, 24, 38]. However, parallel analyses of An. gambiae transcriptional immune responses to $P$. berghei and $P$. falciparum has revealed substantial differences [14] pointing out the necessity to carry out such studies on the human malarial transmission system. Here, we report the first experimental infections of F1 progeny of field caught $A n$. funestus with its natural human parasite P. falciparum.

The study of mosquito susceptibility requires that the technique of infection be able to produce efficient and robust infections in mosquito vectors. In the current study, we tested the use of artificial glass-parafilm membrane system to feed An. funestus mosquitoes with infected blood from gametocyte carriers. This method is clearly preferable to direct feeding assays for ethical reasons, including avoiding the risk of infection of study participants with cryptic pathogens that may be transmitted when using wild-collected mosquitoes. Besides it also offers the possibility to replace the donor's serum with a non-immune $A B$ one to avoid transmission reducing activity of serum components [39].

Nonetheless, the membrane feeding assays present two important weaknesses which could impact the outcome of infection experiments. First, an inappropriate blood handling procedure can result in decline or loss of gametocyte infectivity [40]. Secondly, the number of mosquitoes taking a blood meal could be reduced, especially when using newly collected field mosquito samples, which are not yet adapted to artificial feeding in laboratory conditions [41, 42]. This would explain 


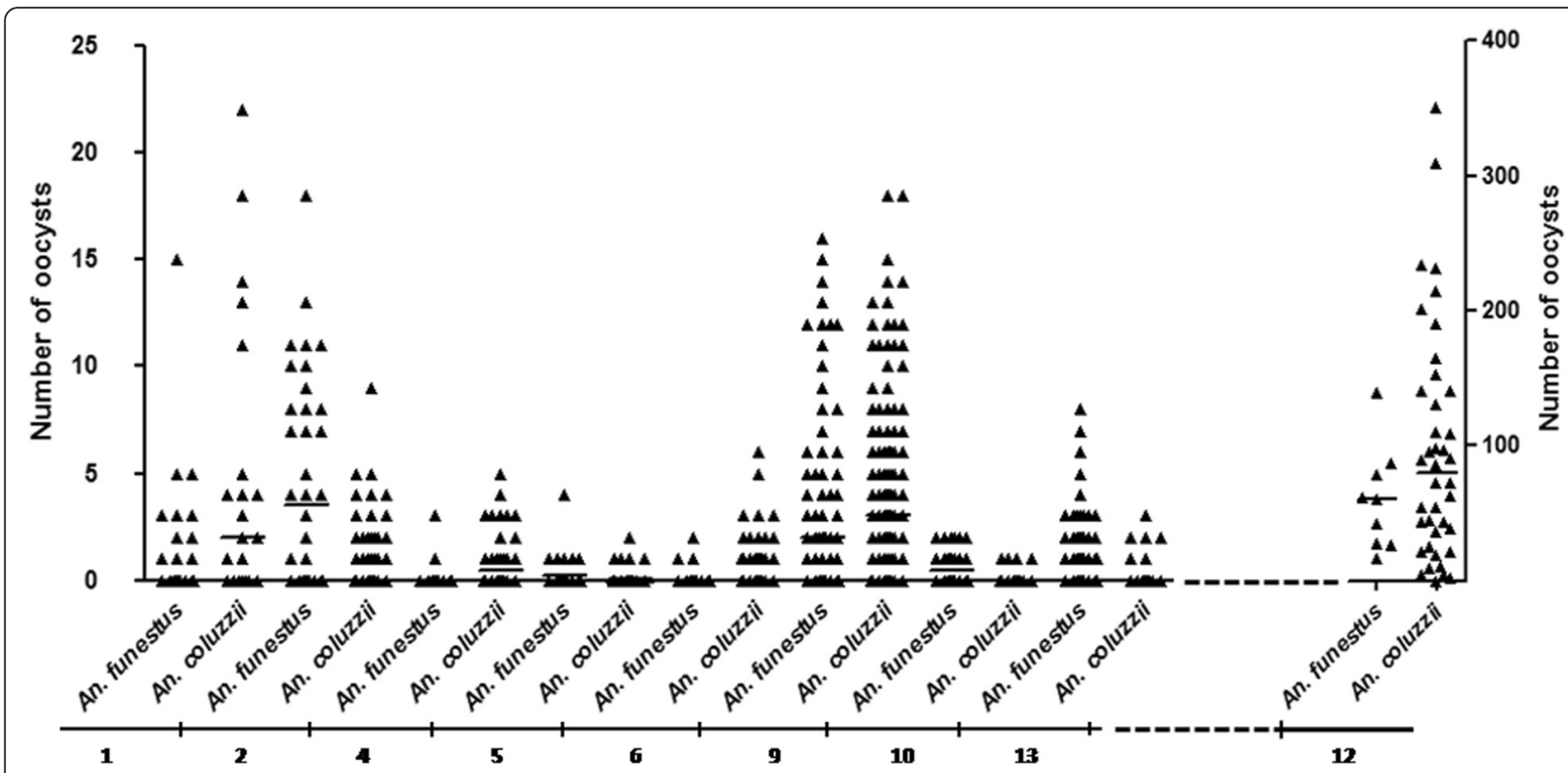

Fig. $\mathbf{2}$ Variation of number of oocyst in individual An. funestus and An. coluzzii midguts in nine experiments. Each triangle represents the number of oocysts in an individual midgut. Dotted lines on the $X$-axis separate experiment 12 from the rest to better see variation in oocyst number in all experiments. The $Y$-axis on the left is related to experiments $1,2,4,5,6,9,10$ and 13 . The $Y$-axis on the right is only related to experiment 12 , for which very high number of oocysts in individual midgut was recorded

the differences in feeding rate observed in this study between the $A n$. coluzzii Ngousso lab strain and the first generation wild An. funestus.

In this study, mosquitoes became infected in the majority (over $75 \%$ ) of experiments demonstrating that the parafilm/glass feeders system and the blood handling procedure used for An. gambaie (s.l.) are also suitable for An. funestus experimental infections. However, the feeding rate of F1females from wild An. funestus mosquitoes varied significantly across experiments and did not exceed $50 \%$ in all cases. This was lower than those previously reported in An. funestus Fumoz laboratory strain (over $50 \%$ ) and in An. gambiae (51.9 \%) [22]. The difference could more likely be due to the use of mice feeding system in the study of Lo \& Coetzee [22] which better mimics the natural feeding condition of Anopheles mosquitoes and/or to a better adaptation of the Fumoz lab strain to feed in lab conditions. By contrast, our results are comparable to those of a similar study conducted in Senegal [42] in which the authors also used the parafilm/glass feeder system to infect wild $A n$. arabiensis F1 progeny and reported low feeding rate $(32.7 \%)$ further confirming that freshly field collected mosquitoes are not well adapted to feed on artificial membrane as stated above. Nonetheless, low feeding rate of wild Anopheles strains could be compensated by increasing the number of mosquitoes for infection experiments and this can now be easily achieved for $A n$. funestus, since collection and rearing of this mosquito species have been significantly improved [15].

The infection parameters (mean prevalence of infection 25.66; median oocyst number 12.5, mean oocyst load 7.02) recorded in this study were higher than that reported in a similar one using the An. funestus insecticide resistant (FUMOZ) and susceptible (FANG) lab strains and $P$. berghei parasite (mean prevalence of infection $20 \%$ ) [22]. Conversely, wild An. funestus genotypes from Mali (West Africa) exhibited significantly higher prevalence of infection (62-97\%) when infected with $P$. berghei [23]. The variations in prevalence of infection between the three studies could be explained by three main factors which have been shown to significantly predict the proportion of mosquitoes that become infected after taking an infectious blood meal: (i) the difference in gametocyte densities in blood used for experimental infections [43]; (ii) the difference in gametocyte sex ratio in blood [44]; and more likely (iii) the difference of infectivity of Plasmodium species used [45]. However, it is noteworthy that $A n$. funestus is a vector of $P$. falciparum and do not naturally interact with $P$. berghei. Therefore, by using natural isolate of the human malaria parasite $P$. falciparum, the present study gives the more realistic picture of its vector competence.

Anopheles funestus displayed high and comparable levels of susceptibility to P. falciparum infection in comparison to An. coluzzii as measured by the percentage of 
experiments showing infected mosquitoes, prevalence of infection and oocyst number in midguts. Our observations reinforce the view that both species are readily infected with $P$. falciparum, the most common and dangerous malaria parasite in sub-Saharan Africa, which may have had a greater ability to develop defense strategies against the mosquito immune system due to their long period of co-evolution [40].

Although our primary objective was not to investigate the kinetics of P. falciparum development in An. funestus, the fact that oocysts were observed in midguts after dissection of mosquitoes at day 7 gives preliminary indication that the parasite develops at similar rates to those in An. gambiae [46]. Further investigations with dissection of mosquitoes at later time points at which sporozoites should be observed in salivary glands are needed.

\section{Conclusion}

This study reported high levels of susceptibility of wild An. funestus progeny to P. falciparum and since this mosquito is widespread throughout Africa, malaria vector control research and implementation need to seriously address this vector species too. Our results also demonstrate the applicability of the parafilm/glass feeding assays for An. funestus experimental infections with its natural parasite $P$. falciparum. This open new avenues toward further investigations of An. funestusPlasmodium interactions in natural system, taking advantage of genomic tools now available.

\section{Additional file}

Additional file 1: Table S1. Infection parameters in An. funestus and An. coluzzii in nine parallel experiments. For each experiment, median oocyst number followed by the same letter are not significantly different; gametocyte densities correspond to the number of gamaetocytes per microliter of blood. (DOCX $16 \mathrm{~kb}$ )

\section{Abbreviations}

ELISA, enzyme-linked immunosorbent assay; IRS, indoor residual house spraying; LLINs, long-lasting insecticide-treated nets; OCEAC, Organisation de Coordination pour la lutte contre les Endémies en Afrique Centrale

\section{Acknowledgements}

We are grateful to the volunteers from Okola and the district hospital of Okola for their participation in this study.

\section{Funding}

This work was supported by a Wellcome Trust Training Fellowship in Public Health and Tropical Medicine (102543/Z/13/Z) to CN. The Wellcome Trust had no role in the design of the study and collection, analysis, and interpretation of data and in writing the manuscript.

\section{Authors' contributions}

Conceived the study: CN, CSW. Designed the study protocol CN, CSW and GL. Participated in field and laboratory analyses: CN, EK, BM, JCT. CN analysed data and drafted the manuscript with contribution from CSW, GL, PAA. All the authors read and approved the final version.

\section{Competing interests}

The authors declare that they have no competing interests.

\section{Ethics approval and consent to participate}

The study was approved by the Cameroonian national ethical committee for research on human health (statement N²015/01/535/CE/NRERSH/SP). The gametocyte carriers used in this study were enrolled as volunteers. Their parents or legal guardians signed a written informed consent, after the procedures of the study were fully explained to them. All of the children found infected with malaria parasites received free antimalarial treatment.

\section{Author details}

${ }^{1}$ Malaria Research Laboratory, Organisation de Coordination pour la lutte contre les Endémies en Afrique Centrale (OCEAC), P.O. Box 288, Yaoundé, Cameroon. ${ }^{2}$ Department of Vector Biology, Liverpool School of Tropical Medicine, Pembroke Place, Liverpool L3 5QA, UK. ${ }^{3}$ Faculty of Medicine and Pharmaceutical Sciences, University of Douala, P.O. Box 2701, Douala, Cameroon. ${ }^{4}$ Faculty of Sciences, University of Yaoundé I, P.O. Box 337, Yaoundé, Cameroon. ${ }^{5}$ Research Unit Liverpool School of Tropical Medicine, OCEAC, P.O. Box 288, Yaoundé, Cameroon

Received: 16 March 2016 Accepted: 7 June 2016

Published online: 14 June 2016

\section{References}

1. WHO. World malaria report 2014. Geneva: World Health Organization; 2014.

2. Fontenille D, Lochouarn L, Diagne N, Sokhna C, Lemasson JJ, Diatta M, et al. High annual and seasonal variations in malaria transmission by anophelines and vector species composition in Dielmo, a holoendemic area in Senegal. Am J Trop Med Hyg. 1997;56:247-53.

3. Fontenille D, Simard F. Unraveling complexities in human malaria transmission dynamics in Africa through a comprehensive knowledge of vector populations. Comp Immunol Microbiol Infect Dis. 2004;27:357-75.

4. Cohuet A, Simard F, Wondji CS, Antonio-Nkondjio C, Awono-Ambene P, Fontenille D. High malaria transmission intensity due to Anopheles funestus (Diptera: Culicidae) in a village of savannah-forest transition area in Cameroon. J Med Entomol. 2004;41:901-5.

5. Lwetoijera DW, Harris C, Kiware SS, Dongus S, Devine GJ, McCall PJ, Majambere S. Increasing role of Anopheles funestus and Anopheles arabiensis in malaria transmission in the Kilombero Valley,Tanzania. Malar J. 2014;13: 331.

6. malERA Consultative Group on Vaccines. A research agenda for malaria eradication: vector control. PLoS Med. 2011;8:e1000401.

7. Giardina F, Kasasa S, Sié A, Utzinger J, Tanner M, Vounatsou P. Effects of vector-control interventions on changes in risk of malaria parasitaemia in sub-Saharan Africa: a spatial and temporal analysis. Lancet glob health. 2014:2:e601-15.

8. Bhatt S, Weiss DJ, Cameron E, Bisanzio D, Mappin B, Dalrymple U, et al. The effect of malaria control on Plasmodium falciparum in Africa between 2000 and 2015. Nature. 2015;526:207-11.

9. Cohuet A, Osta MA, Morlais I, Awono-Ambene PH, Michel K, Simard F, et al. Anopheles and Plasmodium: from laboratory models to natural systems in the field. EMBO Rep. 2006;7:1285-9.

10. Gantz VM, Jasinskiene N, Tatarenkova O, Fazekas A, Macias VM, Bier E, James AA. Highly efficient Cas9-mediated gene drive for population modification of the malaria vector mosquito Anopheles stephensi. Proc Natl Acad Sci USA. 2015;112:6736-43. doi:10.1073/pnas.1521077112.

11. Hammond A, Galizi R, Kyrou K, Simoni A, Siniscalchi C, Katsanos D, et al. A CRISPR-Cas9 gene drive system targeting female reproduction in the malaria mosquito vector Anopheles gambiae. Nat Biotechnol. 2016;34(1):78-83.

12. Blandin SA, Wang-Sattler R, Lamacchia M, Gagneur J, Lycett G, Ning Y, et al. Dissecting the genetic basis of resistance to malaria parasites in Anopheles gambiae. Science. 2009;326:147-50.

13. Osta MA, Christophides GK, Kafatos FC. Effects of mosquito genes on Plasmodium development. Science. 2004;303:2030-32.

14. Dong YM, Aguilar R, Xi ZY, Warr E, Mongin E, Dimopoulos G. Anopheles gambiae immune responses to human and rodent Plasmodium parasite species. PLoS Pathog. 2006;2:513-25.

15. Morgan JC, Irving H, Okedi LM, Steven A, Wondji CS. Pyrethroid resistance in an Anopheles funestus population from Uganda. PLoS One. 2010;5:e11872. 
16. Hunt RH, Brooke BD, Pillay C, Koekemoer LL, Coetzee M. Laboratory selection for and characteristics of pyrethroid resistance in the malaria vector Anopheles funestus. Med Vet Entomol. 2005;9:271-5.

17. Wondji CS, Irving H, Morgan J, Lobo NF, Collins FH, Hunt RH, et al. Two duplicated $\mathrm{P} 450$ genes are associated with pyrethroid resistance in Anopheles funestus, a major malaria vector. Genome Res. 2009;19:452-9.

18. Cuamba N, Morgan JC, Irving H, Steven A, Wondji CS. High level of pyrethroid resistance in an Anopheles funestus population of the Chokwe District in Mozambique. PLoS One. 2010;5(6):e11010.

19. Djouaka R, Irving H, Tukur Z, Wondji CS. Exploring mechanisms of multiple insecticide resistance in a population of the malaria vector Anopheles funestus in Benin. PLoS One. 2011;6:e27760.

20. Mulamba C, Irving H, Riveron JM, Mukwaya LG, Birungi J, Wondji CS. Contrasting Plasmodium infection rates and insecticide susceptibility profiles between the sympatric sibling species Anopheles parensis and Anopheles funestus s.s: a potential challenge for malaria vector control in Uganda. Parasit Vectors. 2014;7:71.

21. Riveron JM, Ibrahim SS, Chanda E, Mzilahowa T, Cuamba N, Irving $H$, et al. The highly polymorphic CYP6M7 cytochrome P450 gene partners with the directionally selected CYP6P9a and CYP6P9b genes to expand the pyrethroid resistance front in the malaria vector Anopheles funestus in Africa. BMC Genom. 2014;15:817.

22. Lo TM, Coetzee M. Marked biological differences between insecticide resistant and susceptible strains of Anopheles funestus infected with the murine parasite Plasmodium berghei. Parasit Vectors. 2013;6:184.

23. Xu J, Hillyer JF, Coulibaly B, Sacko M, Dao A, Niare O, et al. Wild Anopheles funestus mosquito genotypes are permissive for infection with the rodent malaria parasite Plasmodium berghei. PLoS One. 2013;8(4):e61181.

24. Aguilar R, Dong Y, Warr E, Dimopoulos G. Anopheles infection responses: laboratory models versus field malaria transmission systems. Acta Trop. 2005;95:285-91

25. Gillies MT, De Meillon B. The Anophelinae of Africa south of the Sahara. Publ Sth Afr Inst Med Res. 1968:54:1-343.

26. Gillies M, Coetzee M. A supplement to the Anophelinae of Africa South of the Sahara. Publ Sth Afr Inst Med Res. 1987;55:1-143.

27. Koekemoer LL, Kamau L, Hunt RH, Coetzee M. A cocktail polymerase chain reaction assay to identify members of the Anopheles funestus (Diptera: Culicidae) group. Am J Trop Med Hyg. 2002;66:804-11.

28. Cohuet A, Simard F, Toto JC, Kengne P, Coetzee M, Fontenille D. Species identification within the Anopheles funestus group of malaria vectors in Cameroon and evidence for a new species. Am J Trop Med Hyg. 2003;69:200-5.

29. Beier JC, Perkins P, Koros J, Onyango FK, Gargan TP, Wirtz R, et al. Malaria sporozoïte detection by dissection and ELISA to assess infectivity to Afrotropical Anopheles (Diptera: Culicidae). J Med Entomol. 1990;27:377-84.

30. Burkot TR, Williams JL, Schneidier I. Identification of Plasmodium falciparum infected mosquitoes by a double antibody enzyme-linked immunosorbentassay. Am J Trop Med Hyg. 1984;33:783-8.

31. Beier J, Perkkins PV, Wirtz RA, Koros J, Diggs D, Gargan TP, Koech DK. Blood- meal identification by direct-enzyme linked immunosorbent assay (ELISA), tested on Anopheles (Diptera: Culicidae) in Kenya. J Med Entomol. 1988;25:9-16.

32. Boissiere A, Gimonneau G, Tchioffo MT, Abate L, Bayibeki A, AwonoAmbene $\mathrm{PH}$, et al. Application of a GPCR assay in the investigation of susceptibility to malaria infection of the $\mathrm{M}$ and $\mathrm{S}$ molecular forms of A. gambiae s.s. in Cameroon. PloS One. 2013;8:e54820.

33. Ehiolé SP, Bissagnéné E, Girard PM. Mémento thérapeutique du paludisme en Afrique. IMEA-Doin. 2008. p. 99.

34. Bousema JT, Drakeley CJ, Mens PF, Arens T, Houben R, Omar SA, et al. Increased Plasmodium falciparum gametocyte production in mixed infections with P. malariae. Am J Trop Med Hyg. 2008;78:442-8.

35. Nsango SE, Abate L, Thoma M, Pompon J, Fraiture M, Rademacher A, et al. Genetic clonality of Plasmodium falciparum affects the outcome of infection in Anopheles gambiae. Int J Parasitol. 2012;42(6):589-95.

36. Metselaar D, Van Thiel PH. Classification of malaria. Trop Geogr Med. 1959; 11:157-61.

37. Hay SI, Snow RW. The Malaria Atlas Project: developing global maps of malaria risk. PLoS Med. 2006;3:e473.

38. Riehle MM, Xu J, Lazzaro BP, Rottschaefer SM, Coulibaly B, Sacko M, et al. Anopheles gambiae APL1 is a family of variable LRR proteins required for Rel1-mediated protection from the malaria parasite, Plasmodium berghei. PLoS One. 2008:3:e3672.
39. Bousema T, Dinglasan RR, Morlais I, Gouagna LC, Van Warmerdam T, Awono-Ambene $\mathrm{PH}$, et al. Mosquito feeding assays to determine the infectiousness of naturally infected Plasmodium falciparum gametocyte carriers. PLoS One. 2012;7(8):e42821.

40. Hume JC, Tunnicliff M, Ranford-Cartwright LC, Day KP. Susceptibility of Anopheles gambiae and Anopheles stephensis to tropical isolates of Plasmodium falciparum. Malar J. 2007;6:139.

41. Graves PM. Studies on the use of a membrane feeding technique for infecting Anopheles gambiae with Plasmodium falciparum. Trans R Soc Trop Med Hyg. 1980;74:738-42.

42. Awono-Ambene HP, Diawara L, Robert V. Comparison of direct and membrane feeding methods to infect Anopheles arabiensis with Plasmodium falciparum. Am J Trop Med Hyg. 2001;64:32-4.

43. Da DF, Churcher TS, Yerbanga RS, Yaméogo B, Sangaré I, Ouedraogo JB, et al. Experimental study of the relationship between Plasmodium gametocyte density and infection success in mosquitoes; implications for the evaluation of malaria transmission-reducing interventions. Exp Parasitol. 2015;149:74-83

44. Robert V, Read AF, Essong J, Tchuinkam T, Mulder B, Verhave JP, Carnevale $P$. Effect of gametocyte sex ratio on infectivity of Plasmodium falciparum to Anopheles gambiae. Trans R Soc Trop Med Hyg. 1996;90(6):621-4.

45. Collins WE, Sullivan JS, Nace D, Williams T, Sullivan JJ, Galland GG, et al. Experimental infection of Anopheles farauti with different species of Plasmodium. J Parasitol. 2002;88(2):295-8.

46. Ndiath MO, Cohuet A, Gaye A, Konate L, Mazenot C, Faye O, et al. Comparative susceptibility to Plasmodium falciparum of the molecular forms $\mathrm{M}$ and $\mathrm{S}$ of Anopheles gambiae and Anopheles arabiensis. Malar J. 2011;10:269.

\section{Submit your next manuscript to BioMed Central and we will help you at every step:}

- We accept pre-submission inquiries

- Our selector tool helps you to find the most relevant journal

- We provide round the clock customer support

- Convenient online submission

- Thorough peer review

- Inclusion in PubMed and all major indexing services

- Maximum visibility for your research

Submit your manuscript at www.biomedcentral.com/submit
C Biomed Central 